\title{
Towards a better understanding of structure-performance relation in PEMFC fuel cells based on ptychography X-ray nanotomography and scanning small angle X-ray scattering
}

\author{
C. Appel ${ }^{1}$, K. Jeschonek ${ }^{2}$, K. Brunnengräber ${ }^{2}$, B.J.M. Etzold ${ }^{2}$, M. Liebi ${ }^{3,4}$, M. Guizar-Sicairos ${ }^{1}$ \\ ${ }^{1}$ Paul Scherrer Institut - PSI, Villigen-5232 Switzerland, \\ ${ }^{2}$ Technical University of Darmstadt, Darmstadt-64287, Germany, \\ ${ }^{3}$ EMPA - Center of X-ray analytics, St. Gallen-9014 Switzerland, \\ ${ }^{4}$ Chalmers University of Technology, Gothenburg 41296, Sweden
}

\section{Christian.appel@psi.ch}

Advanced composite materials will play a big role to meet growing challenges for future energy solutions. In the transportation sector polymer electrolyte membrane fuel cells (PEMFCs) are promising alternatives to combustion engines to reduce $\mathrm{CO}_{2}$ emissions. PEMFC generate electricity by electrochemical reactions that take place in a complex environment within a so-called catalyst layer. In recent years, fundamental and applied PEMFC research has continuously been trying to improve its understanding of structure-performance relation of these layers. PEMFC catalyst layers are porous materials built from three different components; chemically active sites, electron conducting support and proton conducting binder. This study focusses on investigating Pt nanoparticles on carbon black support (PtC, HiSPEC3000) spray-coated on a polymer membrane (Nafion 211). We investigate three different samples with varying amounts of binder (PTFE) but an equal loading of catalyst $\left(\mathrm{m}_{\mathrm{Pt}}=0.2 \mathrm{mg} / \mathrm{m}^{2}\right)$.

Scanning SAXS was performed at the cSAXS beamline of the Swiss Light Source (SLS) to study the meso and nanoscale of the samples based on simultaneous measurements of X-ray scattering and fluorescence (XRF) spectroscopy from small regions of $10 \times 10 \mu \mathrm{m}^{2}$ within the catalyst layers. Figure 1A shows an exemplarily SAXS curve revealing statistical information of the nanostructure by e.g. evaluating the power law scaling at low $\mathrm{q}$. The corresponding XRF spectroscopy data from XRF is particular interesting due to its ability to measure the distribution of $\mathrm{Pt}$ in the catalyst layer based on the $\mathrm{Pt} \mathrm{M}_{\alpha}$ emission line at $2.05 \mathrm{keV}$. A major advantage of scanning SAXS is also its capability to probe the data illustrated in Figure 1A/B on a macroscopic length scales, to possibly use these two features, power law scaling exponent at low q (Figure 1C) and the intensity of the $\mathrm{Pt}_{\alpha}$ (Figure 1D), to generate 2D scattering maps for a $1 \times 1 \mathrm{~mm}^{2}$ area. Based on the results obtained from these maps, specific areas were chosen to mill out cylindrical $\mu \mathrm{m}$-sized pillars using FIB/SEM (see Figure 1F). These pillars were then investigated with the OMNI setup [1] at cSAXS to reveal the 3D nanostructure with ptychography X-ray nanotomography (PXCT) down to a resolution of $26 \mathrm{~nm}$. Figure 1E shows the rendered pore network from one of the catalyst layer color-coded with the pore size distribution (threshold segmented). Currently, we explore different approaches to correlate the imaging data (PXCT) with the statistical data (SAXS and XRF). Our vision is to obtain 3D representative models for the catalyst layers based on the real structure (3D nanostructure PXCT, resolution 26nm), complemented with statistical data from SAXS and XRF down to single nanometer length scale.

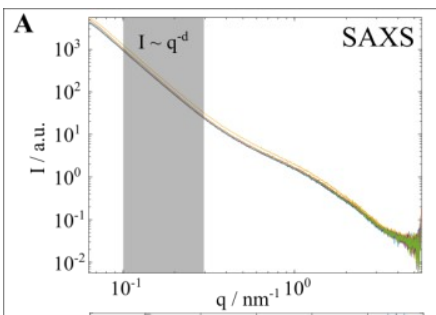

B

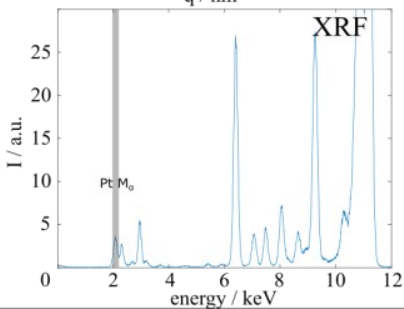

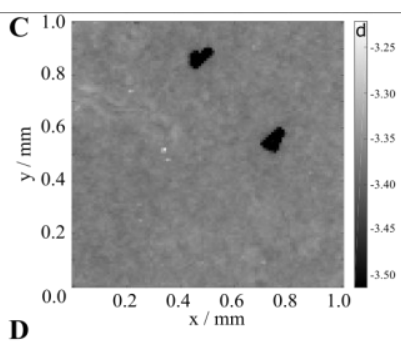

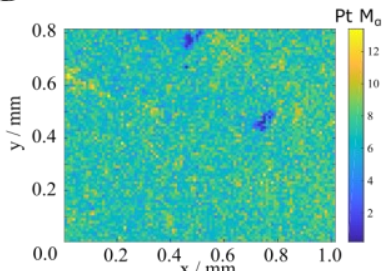

$\mathbf{E}$
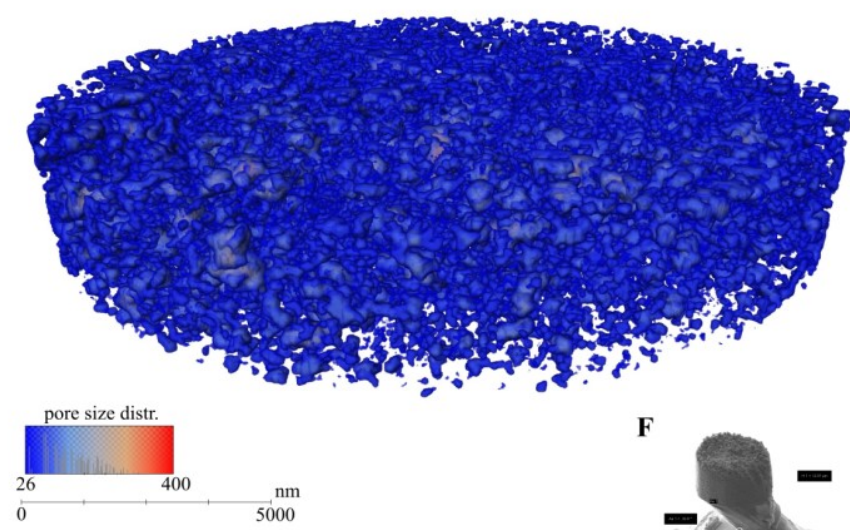

$\mathbf{F}$

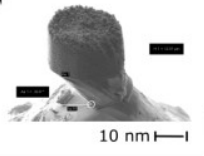

Figure 1. X-ray scattering (A, SAXS), fluorescence (B, XRF) and 2D scattering/fluorescence maps (C, D) of a PEMFC catalyst layer. E 3D rendered pore network measured by PXCT (3D resolution of 26nm), F SEM image of $\mu$-pillar (20 $\mu$ m diameter) of PXCT sample. 
[1] M. Holler, M. Guizar-Sicairos, et. Al. (2017). Rev. Sci. Instrum. 88(11),113701.

\section{Keywords: PXCT; SAXS; PEMFC}

This project has received funding from the European Union's Horizon 2020 research and innovation program under the Marie Sklodowska-Curie grant agreement No 701647 (PSI-FELLOW-III-3i) and funding from the Chalmers initiative for advancement of neutron and $x$-ray techniques. The authors acknowledge the funding from the European Research Council (ERC) under the European Union's Horizon 2020 research and innovation program (grant agreement No. 681719). We also acknowledge the Paul Scherrer Institut, Villigen, Switzerland for provision of synchrotron radiation beamtime at beamline cSAXS of the SLS. 